\title{
Potencial da semeadura direta na restauração florestal de pastagem abandonada no município de Piracaia, SP, Brasil ${ }^{1}$
}

\author{
Andrea Garafulic Aguirre ${ }^{2,6}$, Juliana Toledo Lima ${ }^{3}$, Juliana Teixeira ${ }^{4}$ e Sergius Gandolfi ${ }^{5}$
}

Recebido: 8.04.2015; aceito: 11.09.2015

\begin{abstract}
Potential of direct seeding in forest restoration of an abandoned pasture in the municipal area of Piracaia, São Paulo State, Brazil). The successful establishment of tree species in pastures may be related to the species used, germination conditions, and seed size. The aim of this study was to evaluate the effectiveness of treatments: pre-hydration and different kinds of seed coverage in eight native species with different seed sizes and verify if these species would be able to produce 1,666 individual ha-1 after one year. Five of the eight species tested had potential for large-scale use with the higher germination rate for the small seeds. The pre-hydration of the seeds was not efficient in increasing the germination and in the establishment of species, but coverage with straw and soil showed important results. The best result was obtained with the treatment of seed coverage with soil without hydration, with the establishment of 3,817 seedlings ha ${ }^{-1}$ after one year. Keywords: seed coating, seed pre-hydration, seed size
\end{abstract}

RESUMO - (Potencial da semeadura direta na restauração florestal de pastagem abandonada no município de Piracaia, SP, Brasil). O sucesso do estabelecimento de espécies arbóreas em pastagens pode estar relacionado a espécie utilizada, condições de germinação e tamanho das sementes. O objetivo desse trabalho foi avaliar a eficiência dos tratamentos: pré-hidratação e diferentes coberturas em oito espécies nativas com diferentes tamanhos de sementes e verificar se estas seriam capazes de produzir após um ano 1.666 indivíduos ha ${ }^{-1}$. Cinco espécies testadas apresentaram potencial para uso em grande escala, sendo que as sementes pequenas obtiveram a maior taxa de germinação. A pré-hidratação das sementes não se mostrou eficiente no aumento da germinação e estabelecimento das espécies, mas a cobertura por palha ou terra foi de grande importância. O melhor resultado obtido foi com o tratamento das sementes cobertas com terra e sem hidratação, com estabelecimento de 3.817 mudas ha ${ }^{-1}$ após um ano.

Palavras-chave: pré-hidratação de sementes, recobrimento de sementes, tamanho de sementes

\section{Introdução}

Existindo em toda a região tropical uma grande quantidade de áreas a serem restauradas, a semeadura direta pode ser uma das alternativas promissoras, possibilitando a aceleração do processo de recolonização e sucessão secundária (Cole et al. 2010, Ruiz-Jaen \& Aide 2005).

A semeadura direta vem sendo muito utilizada na recuperação de áreas degradadas, pois apresenta-se como uma técnica viável, devido a sua praticidade, rapidez e baixo custo de implantação (Ferreira et al. 2007). Na América do Sul alguns experimentos obtiveram bons resultados, como os de BonillaMoheno \& Holl (2010), Doust et al. (2006), Ferreira et al. (2009), Isernhagen (2010), Pereira (2012) e Santos et al. (2012).

Algumas das vantagens da semeadura direta são a redução de custos, se comparada ao plantio de mudas devido a inexistência dos mesmos em relação

1. Parte da Dissertação de Mestrado de Andrea Garafulic Aguirre

2. Universidade Estadual Paulista "Julio de Mesquita Filho", Avenida 24-A, 1515, Bela Vista, 13506-900 Rio Claro, SP, Brasil

3. Universidade Estadual de Mato Grosso do Sul, Rodovia Dourados - Itahum, km 12, Centro Universitário de Dourados, Caixa Postal 351, 79804-970 Dourados, MS Brasil

4. Divisão Técnica de Unidade de Conservação, Proteção da Diversidade e Herbário, DEPAVE 8, SVMA/SP - Rua do Paraíso, 387, Paraiso, 04103-000 São Paulo, SP Brasil

5. Universidade de São Paulo, Escola Superior de Agricultura Luiz de Queiroz, Departamento de Botânica, Avenida Pádua Dias, 11, Caixa Postal 9, 13418-900 Piracicaba, SP, Brasil

6. Autor para correspondência: deia.aguirre@gmail.com 
à produção de mudas em viveiro, e o transporte destas ao campo, além da mão-de-obra para abertura de covas e plantio (Doust et al. 2006); apresenta ainda a possibilidade de ser aplicada em áreas de difícil acesso, onde as condições de solo tornam o plantio caro ou impossível (Close \& Davidson 2003). As principais desvantagens seriam as imprevisibilidades das taxas de germinação em campo (Araki 2005); maior manutenção das mudas nos dois primeiros anos pós-germinação, uma vez que estas requerem mais cuidados e tratos culturais adicionais (Araki 2005); a baixa viabilidade de algumas espécies (Lamb 2011); mortalidade elevada das mudas principalmente no primeiro ano (Falck 2005); e eventual carência de micro-sítios adequados para germinação e estabelecimento (De Steven 1991).

Para o sucesso com a técnica é de extrema importância a criação de um micro-sítio com condições favoráveis para uma rápida germinação (De Steven 1991). Esses micro-sítios podem ser estabelecidos com o uso de "mulch" ou de uma camada superficial de terra sobre as sementes para mantê-las úmidas, e para protegê-las da luz solar e de predadores (Sun et al. 1995, Woods \& Elliott 2004, Malavasi et al. 2005). Outro fator importante é o condicionamento osmótico das sementes em campo, já que este método induz uma alteração no estado fisiológico das sementes fazendo com que os processos pré-germinativos ocorram de forma acelerada (Jisha et al. 2012). Durante o condicionamento osmótico ocorrem incrementos no teor de proteínas solúveis e de enzimas específicas, o que proporciona maior concentração de solutos, resultando em um crescimento mais rápido (Smith \& Cobb 1991). A melhoria no desempenho das sementes após o condicionamento osmótico tem sido correlacionada com processos de reparo macromolecular que ocorrem durante o tratamento (Lanteri et al. 1998). Este método tem se mostrado eficiente, podendo proporcionar maior tolerância das sementes ao estresse ambiental (Jisha et al. 2012).

Por fim, um fator de extrema importância para o sucesso da semeadura direta é a escolha das espécies, uma vez que características como o tamanho das sementes utilizadas pode afetar a sua germinação, estabelecimento e sobrevivência das plântulas (Tunjai \& Elliott 2012). Diversos autores sugerem que o sucesso do estabelecimento pode ser influenciado pelo tamanho das sementes das espécies arbóreas utilizadas (Hooper et al. 2002, Doust et al. 2008, Sovu et al. 2010);-sementes maiores têm maior probabilidade de sobrevivência sob condições estressantes, tais como competição, seca, sombreamento e herbívoros, do que sementes menores (Moles \& Westoby 2004). Apesar disso, sementes pequenas de espécies pioneiras são mais comumente usadas na restauração de ecossistemas, pois ocorrem naturalmente nos primeiros estágios sucessionais (Engel \& Parrotta 2001, Balandier et al. 2009).

O objetivo desse trabalho foi avaliar se existe maior porcentagem de estabelecimento das espécies pela técnica de semeadura direta em relação ao tamanho das sementes utilizadas, quando estas são pré-hidratadas e quando as mesmas apresentam algum tipo de cobertura; também foi avaliada se a técnica foi capaz de produzir uma comunidade arbórea com a mesma densidade de árvores normalmente utilizada em plantios de mudas após um ano; e se a restauração seria mais eficiente ao se utilizar apenas os melhores tratamentos ou uma mescla de todos, uma vez que esta mistura de tratamentos pode apresentar diferenças na densidade total de indivíduos por hectare, número de espécies, composição e estrutura da comunidade.

\section{Material e métodos}

O presente estudo foi realizado em um trecho da mata ciliar do rio Cachoeira, município de Piracaia, São Paulo, Brasil $\left(23^{\circ} 00^{\prime} 58^{\prime \prime}-23^{\circ} 00^{\prime} 31^{\prime \prime S}\right.$, $\left.46^{\circ} 17^{\prime} 04^{\prime \prime}-46^{\circ} 16^{\prime} 26^{\prime \prime} \mathrm{W}\right)$. O município apresenta precipitação anual média de $1.440 \mathrm{~mm} \mathrm{ano}^{-1}$, com estação seca entre junho e agosto (Almeida et al. 2011). A temperatura anual média é de $24^{\circ} \mathrm{C}$. O clima da região é do tipo $\mathrm{Cwb}$, ou seja, temperado úmido com inverno seco e verão úmido (Almeida et al. 2011). Predominam na região os latossolos vermelho-amarelos, com fertilidade natural baixa/a média (Embrapa Solos 2006). O trecho de mata ciliar do rio Cachoeira estudado na presente pesquisa possui vegetação caracterizada como área de ecótono entre a Floresta Ombrófila Densa e a Floresta Estacional Semidecidual (Almeida et al. 2011). A região estudada foi desmatada no final do século XIX para o cultivo de café. Em 1970, grandes áreas passaram a ser inundadas para a construção do Sistema Cantareira e as áreas não inundadas passaram a ser ocupadas por reflorestamentos de Pinus, Eucalyptus e pastagens (Oliveira et al. 2008).

O local de estudo encontra-se às margens da represa do rio Cachoeira e é composto por pastagens formadas por duas espécies de gramíneas exóticas, Melinis minutiflora P.Beauv. e Urochloa humidicola (Rendle) Morrone \& Zuloaga. Para a implantação dos 
diversos projetos de restauração no início de 2009, a área do experimento foi cercada e o gado retirado do local.

Para o estudo de semeadura direta priorizaram-se espécies que pudessem promover rápida cobertura da pastagem abandonada, sendo três dessas espécies pioneiras típicas, intolerantes à sombra, Bauhinia forficata Link. (Fabaceae), Croton floribundus Spreng. (Euphorbiaceae), Solanum lycocarpum A.St.-Hil. (Solanaceae) e duas não pioneiras, tolerantes à sombra, mas também de rápido crescimento a pleno sol (secundárias), Erythrina falcata Benth. (Fabaceae) e Ceiba speciosa (A.St.-Hil.) Ravenna (Malvaceae). Para permitir que após o recobrimento inicial ocorresse a continuidade do processo sucessional, foram escolhidas três espécies tolerantes à sombra, de crescimento mais lento e ciclo de vida mais longo, Seguieria langsdorffii Moq. (Phytolaccaceae), Prunus myrtifolia (L.)Urb. (Rosaceae) e Cedrela fissilis Vell. (Meliaceae).

Em relação ao tamanho das sementes, estas foram classificadas, de acordo com sua massa seca média, em sementes pequenas $(0,01-0,099 \mathrm{~g})$, intermediárias (0,1-4,99 g) e grandes (> $5 \mathrm{~g})$ (Doust et al. 2006), sendo calculado o peso pela quantidade de sementes em $1 \mathrm{~kg}$. Todas as espécies escolhidas ocorrem em grande frequência nos fragmentos próximos à área de estudo, sendo as sementes coletadas no mesmo município, a partir de diferentes matrizes para cada espécie. Antes da utilização das sementes em campo, foi executado o teste de germinação em laboratório; para tanto, estas foram desinfetadas com hipoclorito de sódio (2\%) e em seguida lavadas em água corrente. Foram elaboradas quatro repetições de 25 sementes, utilizando-se vermiculita como substrato e não foram feitos tratamentos para a quebra de dormência. A temperatura utilizada foi de $25{ }^{\circ} \mathrm{C}$ e o fotoperíodo foi de $12 \mathrm{~h}$ de luz branca em câmara de germinação tipo B.O.D.

Em campo, os blocos foram estabelecidos em linhas, (medidas em metros quadrados, para facilitar o controle de gramíneas, como recomendado por Araki, 2005). Antes da semeadura, o solo foi descompactado e, a cada três meses, foram feitas roçadas mecânicas nas entrelinhas e capina manual nas linhas de semeadura. Em fevereiro de 2011, foi realizada a primeira manutenção e, para tanto, foram utilizados 50g de NPK 20-5-20 como adubação de cobertura por muda.

Levando-se em consideração que as condições de campo não podem ser completamente controladas e que cada espécie pode responder de forma diferente às condições do micro-sítio local, optou-se por utilizar oito sementes $\mathrm{m}^{-2}\left(21.120\right.$ sementes $\left.\mathrm{ha}^{-1}\right)$, a fim de se obter, após um ano, um mínimo de 1.666 ind ha $^{-1}$, taxa total de sobrevivência de plântulas de aproximadamente $8 \%$ das sementes utilizadas. Para o cálculo de $8 \%$ da taxa de sobrevivência foi feita uma revisão na literatura sobre a germinação em campo de algumas das espécies utilizadas, tendo sido feita uma média da porcentagem de germinação para todas as espécies, tendo-se utilizado como referência os trabalhos de Rolim et al. (2007) e Isernhagem (2010).

As sementes escolhidas foram separadas em dois lotes com igual densidade e metade foi previamente mergulhada em água por $24 \mathrm{~h}$, seguindo a metodologia de Cole et al. (2010), enquanto a outra metade não foi hidratada.

Como fator A, foram consideradas duas condições de pré-tratamento das sementes: com e sem hidratação prévia. Como fator $\mathrm{B}$, foram utilizadas três condições possíveis de cobertura sobre as sementes: sem cobertura, semente recoberta com uma camada de $2 \mathrm{~cm}$ de terra local e semente recoberta com $2 \mathrm{~cm}$ de folhas secas de gramíneas mortas (palha). Portanto, os seguintes tratamentos foram realizados: sementes hidratadas sem recobrimento (testemunha - HSC), sementes hidratadas e cobertura com terra (HT), sementes hidratadas e cobertura com palha (HP), sementes sem hidratação e sem recobrimento (testemunha - SHSC), sementes sem hidratação e cobertura com terra (SHT), sementes sem hidratação e cobertura com palha (SHP). O delineamento utilizado no experimento foi o fatorial com blocos casualizados (DBC).

Desta forma, o experimento foi realizado em oito linhas (blocos ou repetições), sendo que cada uma delas continha seis parcelas (uma para cada tratamento), totalizando-se 48 parcelas. Cada parcela ocupou uma área retangular de $11 \mathrm{~m}^{2}(1 \times 11 \mathrm{~m})$, contendo 88 sementes (11 sementes por espécie), ou seja, oito sementes $\mathrm{m}^{-2}$ (figura 1 ). O número total de sementes utilizadas por espécie foi de 528 , totalizando 4.224 sementes. A área útil do experimento foi de $2.059 \mathrm{~m}^{2}$, praticamente um quinto de hectare.

Para os tratamentos testemunhas (tratamento controle), as sementes foram apenas espalhadas de forma aleatória sobre a superfície. Para os tratamentos com recobrimento das sementes, as mesmas foram alocadas dentro do sulco de forma a estarem bem distribuídas, a fim de evitar uma possível competição, e foram 
recobertas com uma camada de aproximadamente $2 \mathrm{~cm}$ de terra ou palha. A avaliação indireta da germinação foi feita a partir do número de plântulas emergidas das espécies semeadas. Este monitoramento da germinação foi feito a cada 45 dias nos primeiros seis meses, e ao final de um ano da semeadura, tendo sido marcado cada indivíduo germinado.

A fim de verificar quantos indivíduos teriam se estabelecido por tratamento em um hectare após 360 dias de semeadura, foi feita proporção a partir da área total de cada tratamento, que foi de $343,2 \mathrm{~m}^{2}(29,14$ vezes menor do que um hectare). Logo, o número de plântulas estabelecidas por tratamento foi multiplicado por 29,14. Assim sendo, poderia ser discutido em campo se seria necessário empregar um ou mais tratamentos isoladamente ou se um melhor resultado poderia ser obtido combinando-se, simultaneamente, vários desses tratamentos numa mesma área. Para isso, as características das comunidades de plântulas obtidas nas seis condições (duas testemunhas e quatro tratamentos) testadas isoladamente (comunidades A a

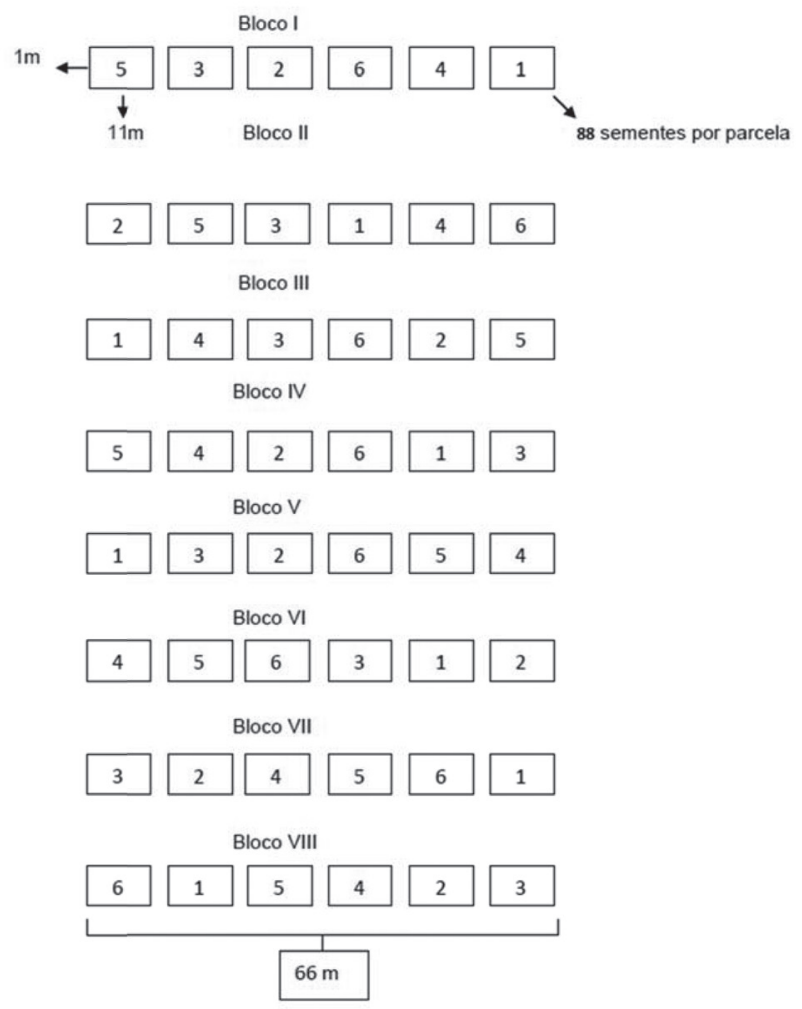

Figura 1 - Esquema de implantação dos tratamentos nas parcelas de acordo com o delineamento experimental em blocos casualizados, pastagem abandonada no município de Piracaia, SP, Brasil.

Figure 1 - Implementation scheme of treatments of treated and untreated installments according to randomized blocks in an abandoned pasture in the of Piracaia, São Paulo State, Brazil.
F), e também as características de duas comunidades obtidas a partir de simulações feitas ao se combinar proporcionalmente os quatro tratamentos mais densos (comunidade $\mathrm{G}=1 / 4 \mathrm{HT}+1 / 4 \mathrm{HP}+1 / 4 \mathrm{SHT}+1 / 4 \mathrm{SHP}$ ) e ao se agrupar proporcionalmente todas as seis condições testadas (comunidade $\mathrm{H}=1 / 6 \mathrm{HSC}+1 / 6$ $\mathrm{HT}+1 / 6 \mathrm{HP}+1 / 6 \mathrm{SHSC}+1 / 6 \mathrm{SHT}+1 / 6 \mathrm{SHP})$. Embora infinitas combinações de tratamentos possam ser analisadas, simulou-se aqui duas comunidades ( $\mathrm{G}$ e $\mathrm{H})$ que hipoteticamente resultariam das combinações de quatro $(\mathrm{G})$ e seis tratamentos $(\mathrm{H})$ dispostos na área degradada de forma proporcional e simultânea. Essas duas comunidades foram simuladas para se observar se alguns de seus atributos poderiam ser mais interessantes para a restauração do que aqueles obtidos nas comunidades isoladas.

Os resultados obtidos em campo para cada tratamento foram analisados estatisticamente por meio de uma análise de variância (ANOVA, um critério), seguido pelo teste de Tukey (Zar 1996) por meio do software BioEstat 5.2 (Ayres et al. 2007). Vale ressaltar ainda que somente as espécies que tiveram mais de $5 \%$ de indivíduos germinados e estabelecidos foram consideradas e incluídas nas análises de comparação dos tratamentos experimentados, sendo elas: Bauhinia forficata, Croton floribundus, Solanum lycocarpum, Erythrina falcata e Ceiba speciosa.

\section{Resultados}

Os resultados referentes aos testes laboratoriais de germinação das sementes mostraram que apenas duas espécies não obtiveram uma boa taxa de germinação, sendo os resultados ordenados de forma decrescente (tabela 1).

Em campo, das 4.224 sementes semeadas, $14,93 \%$ germinaram (631 indivíduos) e dessas $86,84 \%$ conseguiram sobreviver e se estabelecer após o primeiro ano (548 indivíduos). A porcentagem total dos indivíduos estabelecidos variou consideravelmente entre as espécies, sendo que Croton floribundus obteve a maior taxa de germinação $(30,11 \%)$, seguida por Solanum lycocarpum (26,70\%), Bauhinia forficata (18,8\%), Ceiba speciosa (12,87\%) e Erythrina falcata $(12,68 \%)$, enquanto as outras espécies tiveram um estabelecimento abaixo de $5 \%$. Na tabela 2 encontram-se os números de indivíduos de cada espécie que se estabeleceram 360 dias após a semeadura em cada tratamento.

Observou-se que os tratamentos testemunhas (HSC e SHSC) diferiram estatisticamente dos demais tratamentos experimentados $(F=10,8504 ; p>00,5)$. 
Desta forma, obteve-se uma diferença estatística entre os tratamentos onde as sementes foram recobertas e onde não houve a cobertura das mesmas. Todos os tratamentos que tiveram as sementes recobertas, seja por terra ou por palha, não apresentaram diferenças estatisticamente relevantes $(p>0,05)$, mesmo estas sendo pré-hidratadas ou não. Portanto, ao que se refere a pré-hidratação, os resultados mostraram que esta não teve um efeito significativo na germinação e estabelecimento das espécies.

O tratamento de sementes sem hidratação recobertas por terra (SHT) teve o maior número absoluto de plântulas estabelecidas $(131 ; 18,60 \%)$, seguido pelo tratamento de sementes sem hidratação recobertas com palha (SHP, 116; 16,47\%).

A partir da quantidade de plântulas estabelecidas por hectare após um ano, para cada tratamento testado, pode-se verificar que quatro dos seis tratamentos apresentaram média superior a 1.666 mudas ha $^{-1}$, sendo este valor considerado como adequado para a obtenção de cobertura da área em um ano, uma vez que, mesmo se houvesse mortalidade de $50 \%$ das plântulas, ainda sim teríamos 1.666 mudas ha ${ }^{-1}$ após dois anos.

O tratamento das sementes que foram hidratadas, mas não tiveram nenhum tipo de cobertura (SHSC testemunha), apresentou o menor número de sementes estabelecidas (1.223 mudas ha-1), seguido pelo tratamento HSC (testemunha), que apresentou 1.486 mudas $\mathrm{ha}^{-1}$. O melhor tratamento (SHT) resultou em 3.817 mudas $^{-1}$.
A tabela 3 descreve as características das comunidades de plântulas obtidas nos seis tratamentos testados isoladamente (comunidades A a F), além das características de duas comunidades obtidas a partir de simulações feitas ao se combinar proporcionalmente os quatros tratamentos mais densos (comunidade $\mathrm{G})$, e ao se agrupar proporcionalmente todas as seis condições testadas (comunidade $\mathrm{H}$ ).

Em relação à densidade, em plantios feitos com 1.666 mudas ha $^{-1}$, metade dos indivíduos introduzidos (833) deve pertencer a espécies pioneiras e a outra metade a espécies tolerantes à sombra (secundárias); desta forma, todas as comunidades apresentaram após um ano a quantidade adequada de espécies pioneiras e após dois anos (admitindo-se uma mortalidade de 50\% das plântulas estabelecidas), apenas as comunidades A e B não apresentaram resultado adequado, obtendo-se um valor abaixo dos 833 indivíduos por hectare. Já para as espécies secundárias, observa-se que em nenhuma comunidade as densidades obtidas no primeiro ano (87-350 indivíduos $\mathrm{ha}^{-1}$ ) seriam suficientes para formar localmente um segundo dossel contínuo. Quanto à proporção na distribuição das espécies dos dois grupos, podemos considerar que a comunidade "E" foi a mais adequada, uma vez que apresentou a menor porcentagem total de indivíduos pioneiros (79\%) entre todas as comunidades analisadas. Já em relação à riqueza de espécies, das oito comunidades apresentadas, apenas em três $(\mathrm{D}, \mathrm{G}$ e $\mathrm{H})$ todas as espécies semeadas estavam presentes.

Em relação ao tamanho das sementes, pudemos observar que aquelas que tiveram maior taxa de

Tabela 1 - Lista das espécies e respectivas taxas de germinação em laboratório nos lotes de sementes utilizadas no experimento de semeadura direta em pastagem abandonada no município de Piracaia, SP, Brasil. Tamanho das sementes. P: pequeno; M: Médio.

Table 1 - List of species and their laboratory germination rates in seed lots used in direct seeding experiment in an abandoned pasture in the municipal area of Piracaia, São Paulo State, Brazil. Seed size. P: Small; M: Medium.

\begin{tabular}{lcccc}
\hline Nome científico & Nome popular & Tamanho da semente & Massa seca (g) & Média de germinação (\%) \\
\hline Croton floribundus & capixingui & $\mathrm{P}$ & 0,033 & 87 \\
Erythrina mulungu & mulungu & $\mathrm{M}$ & 0,160 & 83 \\
Solanum lycocarpum & fruta-lobo & $\mathrm{P}$ & 0,015 & 79 \\
Ceiba speciosa & paineira & $\mathrm{M}$ & 0,170 & 74 \\
Bauhinia forficata & pata-de-vaca & $\mathrm{M}$ & 0,156 & 71 \\
Cedrela fissilis & cedro-rosa & $\mathrm{P}$ & 0,047 & 55 \\
Seguieria langsdorffii & limão-bravo & $\mathrm{M}$ & 0,160 & 19 \\
Prunus myrtifolia & pessegueiro-bravo & $\mathrm{M}$ & 0,200 & 14 \\
\hline
\end{tabular}


germinação e estabelecimento foram as de tamanho pequeno, Solanum lycocarpum e Croton floribundus; já em relação às sementes médias, as que apresentaram maior taxa de germinação e estabelecimento foram Bauhinia forficata, Ceiba speciosa e Erythrina falcata.

\section{Discussão}

Observou-se que o percentual de estabelecimento por espécie foi abaixo de $30 \%$, sendo que esta taxa também foi encontrada em estudos em áreas agrícolas ou de pastagens abandonadas em áreas tropicais, demonstrando que é comum encontrarmos uma baixa porcentagem de germinação e grandes variações entre as espécies (Ray \& Brown 1995, Sun et al. 1995, Hardwick et al. 1997, Parrotta et al. 1997, Hooper et al. 2002, Woods \& Elliott 2004, Doust et al. 2006, Sampaio 2007). Apesar disso, o número de indivíduos obtidos para a maioria dos tratamentos foi superior ao recomendado para o plantio de mudas em larga escala, o que torna a técnica promissora.

Seguieria langsdorffii e Prunus myrtifolia tiveram baixa viabilidade em laboratório, mostrando que os lotes utilizados possuíam sementes com menor qualidade fisiológica. Além deste fator, as espécies mais tardias da sucessão costumam apresentar redução da germinação e sobrevivência das mudas devido à alta quantidade de luz e condições de temperatura em áreas abandonadas recentemente (Hardwick et al. 1997, Camargo et al. 2002, Vieira \& Scariot 2006).

Cedrela fissilis obteve germinação em laboratório acima de $50 \%$ e no campo abaixo de 5\%. Este resultado pode ser explicado devido ao fato das sementes desta espécie apresentarem um formato alongado e achatado, o que torna mais fácil o contato do embrião com a água, fazendo com que os processos bioquímicos da germinação se apresentem de forma mais rápida e ocorra uma alta taxa de germinação (Liu et al. 2007), mas com baixa tolerância a stress devido aos possíveis processos sucessivos de desidratação (Muller-Landau 2010). Desta forma, a espécie pode ter tido germinação relativamente alta, mas baixo estabelecimento. Embora os resultados para esta espécie não tenham sido animadores, Meneghello \& Mattei (2004) acreditam que ela apresenta um grande potencial na semeadura direta. Uma boa alternativa para otimizar a germinação desta espécie foi encontrada nos estudos realizados por Mattei (1997), Santos Junior et al. (2004) e Klein (2005), que utilizaram protetores físicos os quais ocasionaram uma eficiente germinação. $\mathrm{O}$ uso destes protetores reduz a taxa de herbivoria e promove o aumento da temperatura e umidade da camada superficial do solo (Santos Junior et al. 2004, Ferreira et al. 2007). Apesar do baixo desempenho das três espécies citadas acima, é interessante ressaltar que todas elas germinaram

Tabela 2 - Número total de plântulas estabelecidas por tratamento aos 360 dias após a semeadura em pastagem abandonada no município de Piracaia, SP, Brasil. HSC: sementes hidratadas sem cobertura (testemunha); HT: sementes hidratadas recobertas com terra; HP: sementes hidratadas recobertas com palha; SHSC: sementes sem hidratação e sem cobertura (testemunha); SHT: sementes sem hidratação recobertas com terra; SHP: sementes sem hidratação recobertas com palha.

Table 2 - Total number of established seedlings in each treatment after 360 days in an abandoned pasture in the municipal area of Piracaia, São Paulo State, Brazil. HSC: hydrated seeds without cover (control treatment); HT: hydrated seeds covered with soil; HP: hydrated seeds covered with straw; SHSC: seeds without hydration and without cover (witness); SHT: nonhydrated seeds covered with soil; SHP: non-hydrated seeds covered with straw.

\begin{tabular}{|c|c|c|c|c|c|c|c|}
\hline \multirow[t]{2}{*}{ Espécies } & \multirow{2}{*}{$\begin{array}{l}\text { Sementes por } \\
\text { tratamento }\left(\mathrm{N}^{\circ}\right)\end{array}$} & \multicolumn{6}{|c|}{$\begin{array}{l}\text { Plântulas estabelecidas por tratamento } \\
\qquad\left(\mathrm{N}^{\circ}\right)\end{array}$} \\
\hline & & HSC & HT & HP & SHSC & SHT & SHP \\
\hline Cedrela fissilis & 88 & 0 & 1 & 2 & 0 & 1 & 2 \\
\hline Bauhinia forficata & 88 & 3 & 16 & 16 & 7 & 26 & 28 \\
\hline Solanum lycocarpum & 88 & 20 & 30 & 20 & 10 & 38 & 23 \\
\hline Seguiera langdorffii & 88 & 0 & 1 & 1 & 0 & 0 & 3 \\
\hline Prunus myrtofolia & 88 & 0 & 4 & 0 & 0 & 2 & 0 \\
\hline Erythrina mulungu & 88 & 5 & 15 & 15 & 5 & 13 & 14 \\
\hline Ceiba speciosa & 88 & 5 & 13 & 12 & 6 & 13 & 19 \\
\hline Croton floribundus & 88 & 18 & 25 & 37 & 14 & 38 & 27 \\
\hline Total de indivíduos & 704 & 51 & 105 & 103 & 42 & 131 & 116 \\
\hline
\end{tabular}


apenas nas parcelas onde houve o tratamento de cobertura das sementes.

Para as outras espécies utilizadas, a diferença entre o número de indivíduos germinados em campo e em laboratório pode ter ocorrido por fatores como a predação de sementes, uma vez que o ataque de formigas é preocupante na germinação e na fase de crescimento inicial (Mattei 1997). Além disso, existe a competição com gramíneas, abaixo do solo (Holl 1999, Hooper et al. 2002) e na parte aérea também há competição com herbáceas ruderais, ervas e arbustos que podem normalmente ser comuns em áreas recém abandonadas (Holl 1998), o que tem se mostrado como fator negativo sobre a performance das mudas. Outro fator importante que pode ter prejudicado o estabelecimento das espécies está relacionado com os solos utilizados como pastagens, que em geral, possuem baixa fertilidade natural, acidez elevada e má drenagem, apresentando baixa capacidade de suprir nutrientes, o que resulta em baixos níveis de produtividade (Queiroz et al. 2008).

As espécies que obtiveram maior estabelecimento em nosso experimento foram testadas por Isernhagen
(2010), que obteve taxa de germinação em campo para Croton floribundus de 5\%, Ceiba speciosa apresentou $10,6 \%$ e Solanum lycocarpum apenas 4\%. Já Rolim et al. (2007), para a espécie Bauhinia forficata, obteve média de germinação de $9,55 \%$, entre os três tratamentos utilizados. Desta forma, no geral obtivemos uma maior taxa de estabelecimento, que pode estar associada à qualidade do lote de sementes, assim como pelo recobrimento das mesmas em campo.

Em relação ao tamanho das sementes, as que apresentaram menor porte obtiveram maior estabelecimento quando comparadas com as de médio porte, o que pode estar relacionado às taxas de crescimento rápido de sementes pequenas das espécies em questão; e, com isso, maiores vantagens quando em alta luminosidade, o que é definido como uma estratégia aquisitiva (Wrigth et al. 2010). Baroloto \& Goldberg. (2005) e Pereira (2012) encontraram em seus estudos que as sementes pequenas tendem a ter um maior potencial de crescimento quando não existe uma forte competição com gramíneas.

Utilizar sementes pequenas na restauração ecológica pode ter muitas vantagens porque estas

Tabela 3 - Comunidades de plântulas formadas pela semeadura direta em pastagem abandonada no município de Piracaia, SP, Brasil. $\mathrm{G}=\mathrm{C} 4$ : combinação dos quatro tratamentos com maiores densidades absolutas $(1 / 4 \mathrm{HT}+1 / 4 \mathrm{HP}+1 / 4$ SHT $+1 / 4$ SHP); H = C6: combinação de todas as seis condições testadas (1/6 HSC + 1/6 HT + 1/6 HP + 1/6 SHSC + 1/6 SHT + 1/6 SHP).

Table 3 - Plant communities formed by the direct sowing in abandoned pasture in an the municipal area of Piracaia, São Paulo State, Brazil. G $=\mathrm{C} 4$ : combination of the four treatments with the highest absolute densities $((1 / 4 \mathrm{HT}+1 / 4 \mathrm{HP}+1 / 4$ SHT $+1 / 4$ SHP); H $=$ C6: combination of all six tested conditions $(1 / 6 \mathrm{HSC}+1 / 6 \mathrm{HT}+1 / 6 \mathrm{HP}+1 / 6 \mathrm{SHSC}+1 / 6 \mathrm{SHT}+1 / 6 \mathrm{SHP})$.

\begin{tabular}{lcccccccc}
\hline & $\mathrm{A}$ & $\mathrm{B}$ & $\mathrm{C}$ & $\mathrm{D}$ & $\mathrm{E}$ & $\mathrm{F}$ & $\mathrm{G}$ & $\mathrm{H}$ \\
\cline { 2 - 9 } Espécies & SHSC & HSC & HP & HT & SHP & STH & C4 & C6 \\
\cline { 2 - 9 } & Ind. & Ind. & Ind. & Ind. & Ind. & Ind. & Ind. & Ind. \\
\hline Croton floribundus & 408 & 525 & 1.078 & 729 & 787 & 1.107 & 925 & 772 \\
Solanum lycocarpum & 291 & 583 & 583 & 874 & 670 & 1.107 & 809 & 685 \\
Bauhinia forficate & 204 & 87 & 466 & 466 & 816 & 758 & 627 & 466 \\
Erythrina mulungu & 146 & 146 & 437 & 437 & 408 & 379 & 415 & 325 \\
Ceiba speciosa & 175 & 146 & 350 & 379 & 554 & 379 & 415 & 330 \\
Prunus myrtofolia & 0 & 0 & 0 & 117 & 0 & 58 & 44 & 29 \\
Seguiera langsdorffii & 0 & 0 & 29 & 29 & 87 & 0 & 36 & 24 \\
Cedrela fissilis & 0 & 0 & 58 & 29 & 58 & 29 & 44 & 29 \\
Densidade Total por hectare (espécies 1-8) & 1.224 & 1.486 & 3.001 & 3.060 & 3.380 & 3.817 & 3.315 & 2.661 \\
Densidade Total (pioneiras, 1-4) & 1.049 & 1.340 & 2.564 & 2.506 & 2.681 & 3.351 & 2.776 & 2.249 \\
Densidade Total (secundárias, 5-8) & 175 & 146 & 437 & 554 & 699 & 466 & 539 & 413 \\
50\% da densidade total & 612 & 743 & 1.501 & 1.530 & 1.690 & 1.909 & 1.428 & 1.331 \\
50\% da densidade total (pioneiras, 1-4) & 525 & 670 & 1.282 & 1.253 & 1.340 & 1.676 & 1.206 & 1.124 \\
50\% da densidade total (secundárias, 5-8) & 87 & 73 & 219 & 277 & 350 & 233 & 222 & 206 \\
Número de espécies & 5 & 5 & 7 & 8 & 7 & 7 & 8 & 8 \\
\hline
\end{tabular}


são produzidas em grandes quantidades e são mais facilmente transportadas, o que favorece a dispersão (Obeso et al. 2011); uma vez que sementes menores tendem a permanecer por mais tempo no trato digestivo dos animais, podem ser dispersas para longe da planta-mãe (Obeso et al. 2011) e podem ser ingeridas e defecadas por uma grande variedade de dispersores (Holbrook \& Loiselle 2009). Além disso, sementes pequenas permitem a adoção da técnica de peletização (Lopes \& Nascimento 2012), o que pode aumentar a rapidez e eficiência do plantio (Silva et al. 2002).

Em relação aos diferentes tratamentos experimentados, demonstrou-se que houve diferença significativa dos tratamentos testemunhas, sem hidratação e sem cobertura (SHSC) e hidratado e sem cobertura (HSC) em relação aos demais, sendo estes os únicos que tiveram uma baixa taxa de estabelecimento das espécies. Este resultado mostra que a cobertura das sementes é de fundamental importância para maior germinação e estabelecimento das plântulas das espécies testadas. Este fator já havia sido verificado por alguns estudos, onde as sementes que foram enterradas sofreram uma diminuição

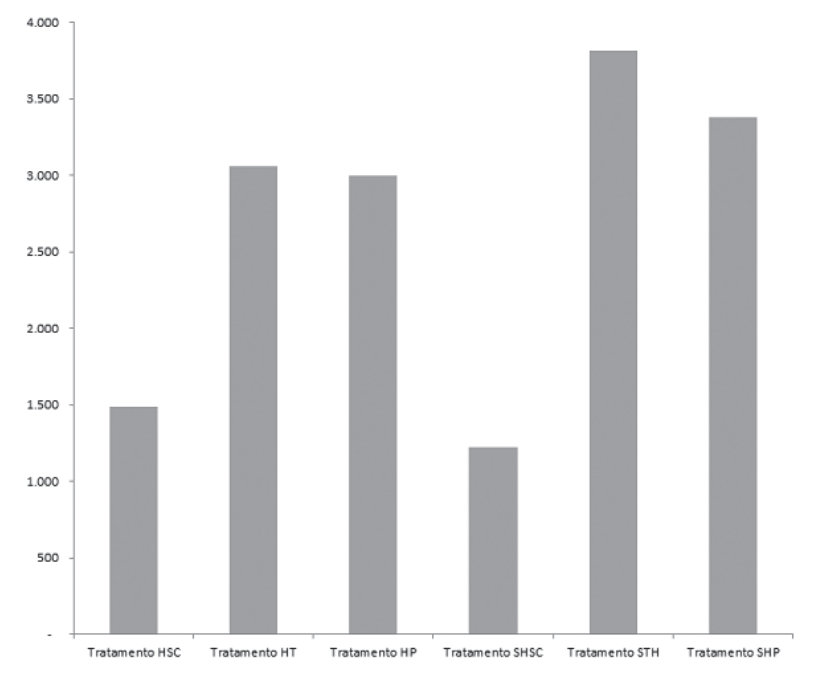

Figura 2 - Quantidade de plântulas estabelecidas por hectare após um ano, para cada tratamento testado em pastagem abandonada no município de Piracaia, SP, Brasil. HSC: sementes hidratadas sem cobertura (testemunha); HT: sementes hidratadas recobertas com terra; HP: sementes hidratadas recobertas com palha; SHSC: sementes sem hidratação e sem cobertura (testemunha); SHT: sementes sem hidratação recobertas com terra; SHP: sementes sem hidratação recobertas com palha.

Figure 2 - Number of established seedlings per hectare after one year for each treatment in an abandoned pasture in the municipal area of Piracaia, São Paulo State, Brazil. HSC: hydrated seeds without soil cover (witness); HT: hydrated seeds covered with earth; HP: hydrated seeds covered with straw; SHSC: seeds without hydration and without cover (witness); SHT: non-hydrated seeds covered with soil; SHP: non-hydrated seeds covered with straw dos efeitos da predação (Woods \& Elliott 2004, Araki 2005, Doust et al. 2006). Neste estudo pudemos verificar que não houve diferença estatística significativa para os tratamentos nos quais as sementes foram recobertas com palha ou terra, ressaltando que, mais importante que o material utilizado, é que as sementes apresentem algum tipo de cobertura.

Apesar de o condicionamento osmótico acelerar a germinação e reduzir o tempo de emergência, não houve diferenças significativas entre os tratamentos onde as sementes foram pré-hidratadas e onde não foram (para os tratamentos que tiveram cobertura de suas sementes). Este resultado corrobora com o de Missanjo et al. (2013), os quais realizaram um experimento com a superação de dormência de Albizia lebbeck e obtiveram como resultado que o método de água fria não foi muito eficiente. Segundo estes mesmos autores, o tegumento foi suavizado, mas o período de $24 \mathrm{~h}$ no qual a semente foi mergulhada em água não foi suficiente para maximizar a porcentagem de germinação. No entanto, a pré-hidratação pode ser um tratamento eficiente se as sementes permanecerem mergulhadas por um tempo acima de 48 h (Likoswe et al., 2008). Este mesmo resultado foi comprovado por Ferrandis et al. (2011) para a espécie Podocarpus angustifolius, onde a taxa de germinação foi acelerada com a utilização da imersão das sementes em água como pré-tratamento por $48 \mathrm{~h}$.

Em alguns casos de sementes recalcitrantes, tem sido demonstrado que o embrião requer um alto nível de hidratação para poder germinar após a dispersão de sementes, sendo a abundância de chuvas necessária para o aumento da resposta de germinação (Baskin $\&$ Baskin 1998). No caso do período de 24 horas, o que pode ocorrer é que a umidade não é suficiente para desencadear o processo de germinação, uma vez que, ao se colocar as sementes em um ambiente com um baixo teor de umidade e grandes temperaturas, estas podem acabar perdendo água por osmose e desta forma podem entrar numa fase de repouso até que a umidade no solo se recupere (Vázquez et al. 1997). Outro ponto importante é que, em algumas espécies, o excesso de umidade pode prejudicar a germinação, principalmente quando estas são atacadas por fungos ou estão em um habitat não favorável à germinação, apesar de estarem hidratadas. Desta forma, a porcentagem de umidade nas sementes já foi mencionada como indicativo da maturidade de várias espécies (Tewari et al. 2011), mas em alguns casos, como o da cereja-selvagem (Prunus cerasoides), a germinação e a umidade contida nas sementes foram negativamente correlacionadas (Tewari et al. 2011). Borges \& Rena (1993) encontraram em seus resultados 
que o excesso de umidade prejudicou a germinação das sementes de pau-d'alho (Gallesia integrifolia), impedindo a penetração de oxigênio e reduzindo o processo metabólico.

A maior densidade total obtida por hectare após um ano pode ser considerada o melhor resultado, pois uma maior densidade permitiria maior e mais rápido sombreamento da área e, consequentemente, menores custos de restauração, que neste caso, seria a condição SHT (tabela 3, comunidade $\mathrm{F}=3.817$ indivíduos ha $^{-1}$ ). Quando se faz plantios usando-se o modelo de preenchimentos e diversidade (Rodrigues et al. 2009), normalmente utilizam-se 1.666 indivíduos ha ${ }^{-1}$, dos quais metade, ou seja, 833 indivíduos ha ${ }^{-1}$, serão os principais responsáveis pela rápida formação de um dossel e o recobrimento inicial da área, o que ocorre em torno de dois anos, partindo da premissa que a restauração é feita de forma correta; isto significa que a área deveria receber um bom preparo prévio, a escolha correta das espécies e uma manutenção adequada (Davide et al. 2000, Rodrigues et al. 2009). Os demais indivíduos, tendo crescimento mais lento, serão responsáveis principalmente pela manutenção futura da floresta. Simulando-se então que, ao longo do segundo ano, $50 \%$ dos indivíduos pioneiros obtidos no primeiro ano viessem a morrer, pode-se novamente avaliar quais comunidades teriam ainda pelo menos os 833 indivíduos pioneiros por hectare necessários ao recobrimento da área ao fim do segundo ano. Neste caso, apenas as comunidades A e B não alcançariam o mínimo necessário. Todavia, este critério não pode ser o único a ser considerado na seleção de qual seria a melhor solução a ser aplicada.

Sabendo-se que as árvores das espécies pioneiras após algum tempo irão morrer, é desejável que a morte desses indivíduos não seja simultânea, para que não haja uma perda sincrônica de todas as copas que formam o dossel inicial, o que poderia levar ao fracasso da restauração (Rodrigues et al. 2009). Assim, se cada espécie pioneira empregada tiver um ciclo de vida distinto, então seria vantajoso obter-se uma comunidade em que as diferentes espécies pioneiras tenham densidades mais proporcionais entre si. Desta forma, a comunidade "E" foi a que apresentou a menor porcentagem total de indivíduos pioneiros (79\%) entre todas as comunidades analisadas, indicando uma melhor distribuição de indivíduos entre os dois grupos semeados.

Em relação às espécies secundárias, nenhuma comunidade conseguiu alcançar, no primeiro ano, 833 indivíduos. Portanto essa semeadura, por si só, não seria capaz de garantir a permanência do habitat florestal após cerca de 20 anos. A restauração ficaria dependente das condições locais de enriquecimento natural, que pudessem aí introduzir mais espécies tardias, ou de uma introdução ativa de mais indivíduos secundários nos anos seguintes à semeadura direta. Como a área de estudo apresenta uma floresta em estágio médio de regeneração, a 500 metros de distância, a chuva de sementes na área poderá suprir este problema.

A composição e estrutura das comunidades obtidas são também aspectos que podem ser relevantes para a escolha do tipo de semeadura a ser escolhido. Das oito comunidades apresentadas, em três (D, G e $\mathrm{H})$ se obteve a máxima riqueza (oito espécies); em outras três $(\mathrm{C}, \mathrm{E}$ e $\mathrm{F})$, uma espécie não germinou, obtendo-se sete espécies; e em outras duas (A e B), apenas cinco espécies germinaram. Desta forma, se a maior biodiversidade obtida for considerada um critério fundamental, então apenas três comunidades deveriam ser escolhidas.

A comunidade $\mathrm{G}$ apresentou, em valores absolutos, maior densidade total (3.315 indivíduos $\mathrm{ha}^{-1}$ ) do que as obtidas com os tratamentos HP $(\mathrm{C}=3.001)$ e HT $(\mathrm{D}=3.060)$; e menor do que as obtidas com SHP e SHT $(\mathrm{E}=3.380$ e $\mathrm{F}=3.817)$. Também apresentou oito espécies, como o tratamento HT (D), e uma espécie a mais que os tratamentos HP, SHP e SHT (respectivamente, comunidades C, E e F). A comunidade $\mathrm{H}$ apresentou valores absolutos de densidade intermediários e a riqueza máxima; mesmo se considerarmos a mortalidade de $50 \%$ das espécies pioneiras após dois anos, ainda teríamos um número considerável de espécies pioneiras, portanto trata-se de uma opção interessante a se considerar na restauração de áreas degradadas.

Tanto a composição de espécies obtidas quanto a estrutura variaram entre as comunidades testadas e simuladas. Esses aspectos podem ser ecologicamente relevantes na dinâmica das comunidades formadas e podem ser critérios também utilizáveis na escolha do método a ser empregado. Uma vez que, devido ao grande número de variáveis ambientais que podem interferir no comportamento das espécies em determinado sítio (Martins 2001), a escolha de um modelo adequado é essencial para o sucesso da restauração. Desta forma, devemos conhecer o comportamento e as exigências de cada espécie para acelerar a implantação de reflorestamentos e levar em consideração que cada método funciona sob um conjunto de condições adequadas e particulares, que 
nem sempre podem ser expressas em outras épocas e lugares (Martins 2001); assim sendo, a utilização de vários tratamentos ao mesmo tempo torna-se útil para a restauração de ecossistema de degradados e para a sua maior capacidade de resiliência.

\section{Conclusões}

Dos seis tratamentos implantados, quatro obtiveram densidade adequada de indivíduos após um ano e, das oito espécies testadas, cinco apresentam um potencial para a utilização na semeadura direta (Croton floribundus, Solanum lycocarpum, Bauhinia forficata, Ceiba speciosa e Erythrina falcata). Constatou-se que, aparentemente, a pré-hidratação não melhorou o desempenho na germinação das espécies de forma geral. Em contrapartida, o recobrimento das sementes foi de fundamental importância para o aumento na germinação e estabelecimento das espécies, independentemente do tipo de recobrimento (terra e palha). Verificou-se ainda que sementes pequenas podem ter um desempenho aceitável na técnica e podem ser úteis para a colonização das áreas restauradas. Por fim, outros fatores extrínsecos (luz, competição e temperatura), assim como fatores intrínsecos (impermeabilidade do tegumento, imaturidade fisiológica e presença de substâncias inibidoras) podem atuar sobre cada espécie de forma distinta, portanto a utilização de vários tratamentos na mesma área é recomendada para que se obtenha maior diversidade e densidade para a técnica. Em linhas gerais, os resultados aqui apresentados demonstraram que a semeadura direta é uma técnica promissora para a restauração em grande escala.

\section{Literatura citada}

Almeida, A., Padovezi, A. \& Lima, R.A.F. 2011. Levantamento e avaliação de informações de referencia para o Projeto de Restauração de 350 hectares do entorno do reservatório do Rio Cachoeira - Piracaia, São Paulo. The Nature Conservancy do Brasil 2: 40-49.

Araki, D.F. 2005. Avaliação da semeadura a lanço de espécies florestais nativas para recuperação de áreas degradadas. Dissertação de Mestrado, Universidade de São Paulo, Piracicaba.

Ayres, M., Ayres Jr.M., Ayres, D.L. \& Santos, A.A. 2007. Bioestat 5.0. Ong Mamirauá, Belém.

Balandier, P., Frochot, H. \& Sourisseau, A. 2009. Improvement of direct tree seeding with cover crops in afforestation: microclimate and resource availability induced by vegetation composition. Forest Ecology and Management 257: 1716-1724.
Baroloto, C.P.M. \& Goldberg, D.E. 2005. Seed mass, seedling size, and neotropical tree seedling establishment. Journal of Ecology 93: 1156-1666.

Baskin, C.C. \& Baskin, J.M. 1998. Seeds: Ecology, biogeography, and evolution of dormancy and germination. Academic Press, San Diego, California.

Bonilla-Moheno, M. \& Holl, K.D. 2010. Direct seeding to restore tropical mature-forest species in areas of slashand-burn agriculture. Restoration Ecology 18: 438-445.

Borges, E.E.L. \& Rena, A.B. 1993. Germinação de sementes. In: I.B. Aguiar, F.C.M. Piña-Rodrigues \& M.B. Figliolia (coords.). Sementes Florestais Tropicais. Abrates, Brasília, pp. 83-135.

Camargo, J.L., Kossman-Ferraz, I.D. \& Imakawa, A.M. 2002. Rehabilitation of degraded areas of Central Amazonia using direct sowing of forest tree seeds. Restoration Ecology 10: 636-644.

Close D.C. \& Davidson, N.J. 2003. Revegetation to combat tree decline in the Midlands and Derwent Valley lowlands of Tasmania: Practices for improved plant establishment. Ecological Management \& Restoration 4: 29-36.

Cole, R.J., Holl, K.D., Keene, C.L. \& Zahawi, R.A. 2010. Direct seeding of late-sucessional tress to restore tropical montane forest. Forest Ecology and Management 261: 1590-1597.

Davide, A.C., Ferreira, R.A., Faria, J.M.R., Botelho, S.A. 2000. Restauração de matas ciliares. Informe Agropecuário 21: 65-74.

De Steven, D. 1991. Experiments on mechanisms of tree establishment in old-field succession: seedling emergence. Ecology 72: 1066-1075.

Doust, S.J., Erskine, P.D. \& Lamb, D. 2006. Direct seeding to restore rainforest species: Microsite effects on the early establishment and growth of rainforest tree seedlings on degraded land in the wet tropics of Australia. Forest Ecology and Management 234: 333-343.

Doust, S.J., Erskine, P.D. \& Lamb, D. 2008. Restoring rainforest species by direct seeding: tree seedlings establishment and growth performance on degraded land in the wet tropics of Australia. Forest Ecology and Management 256: 1178-1188.

Engel, V.L. \& Parrotta, J.A. 2001. An evaluation of direct seeding for reforestation of degraded lands in central Sao Paulo state, Brazil. Forest Ecology and Management 152: 169-181.

Embrapa Solos. 2006. Sistema Brasileiro de Classificação de Solos. 2 ed. Embrapa Solos, Rio de Janeiro.

Falck.G.L. 2005. Recobrimento de sementes de Pinus elliottii Engelm como alternativa para semeadura direta em campo. Dissertação de Mestrado, Universidade Federal de Pelotas, Pelotas. 
Ferrandis, P., Bonilla, M. \& Osorio, L.D.C. 2011. Germination and soil seed bank traits of Podocarpus angustifolius (Podocarpaceae): an endemic tree species from Cuban rain forests. Revista de Biologia Tropical 59: 1061-1069.

Ferreira, R.A., Davide, A.C., Bearzoti, E. \& Motta, M.S. 2007. Semeadura direta com espécies arbóreas para recuperação de ecossistemas florestais. Revista Cerne 13: 21-279.

Ferreira, R.A., Santos, P.L., Aragão, A.G., Santos, T.I.S., Santos Neto, E.M. \& Rezende, A.M.S. 2009. Semeadura direta com espécies florestais na implantação de mata ciliar no Baixo São Francisco em Sergipe. Scientia Forestalis 37: 37-46.

Hardwick, K., Healey, J., Elliott, S., Garwood, N. \& Anusarnsunthorn, V. 1997. Understanding and assisting natural regeneration processes in degraded seasonal evergreen forests in northern Thailand. Forest Ecology and Management 99: 203-214.

Hooper, E., Condit, R. \& Legendre, P. 2002. Responses of 20 native tree species to reforestation strategies for abandoned farmland in Panama. Ecological Applications 12: 1626-1641.

Holbrook, K.M. \& Loiselle, B.A. 2009. Dispersal in a neotropical tree, Virola flexuosa (Myristicaceae): does hunting of large vertebrates limit seed removal? Ecology 90: 1449-1455.

Holl, K.D. 1998. Effects of above-and below-ground competition of shrubs and grass on Calophyllum brasiliense (Camb.) seedling growth in abandoned tropical pasture. Forest Ecology nad Management 109: 187-195.

Holl, K.D. 1999. Factors limiting tropical rain forest regeneration in abandoned pasture: seed rain, seed germination, microclimate, and soil. Biotropica 31: 229-242.

Isernhagen, I. 2010. Uso de semeadura direta de espécies arbóreas ativas para restauração florestal de áreas agrícolas, sudeste do Brasil. Dissertação de Mestrado, Universidade do Estado de São Paulo, Piracicaba.

Jisha, K.C., Vijayakumari, K. \& Jos, T.P. 2012. Seed priming for abiotic stress tolerance: an overview. Acta Physiologiae Plantarum 35: 1381-1396.

Klein, J. 2005.Utilização de protetores físicos na semeadura direta de timburi e canafístula na revegetação de matas ciliares. Dissertação de Mestrado, Universidade Estadual do Oeste do Paraná, Marechal Candido Rondon.

Lamb, D. 2011. Ecological restoration. In: D. Lamb (ed.). Regreening the bare hills: tropical forest restoration in the Asia-Pacific region. Springer, New York, pp. 325-355.

Lanteri, S., Quagliotti, L. \& Belletti, P. 1998. Delayed luminescence and priming-induced nuclear replication of unaged and controlled deteriorated pepper seeds (Capsicum annuum L.). Seed Science and Technology 26: 413-424.
Likoswe, M.G., Njoloma, J. P., Mwase, W.F. \& Chilima, C.Z. 2008. Effect of seed collection times and pretreatment methods on germination of Terminalia sericea Burch. ex DC. African Journal of Biotechnology 7: 2840-2846.

Liu, Z., Yan, Q., Li, X., Ma, J. \& Ling, X. 2007. Seed mass and shape, germination and plant abundance in a desertified grassland in northeastern Inner Mongolia, China. Journal of Arid Environments 69: 198-211.

Lopes, A.C.A. \& Nascimento, W.M. 2012. Peletização de sementes de hortaliças. Embrapa hortaliças, Brasilia, DF.

Malavasi, U.C., Gasparino, D. \& Malavasi, M.M. 2005. Semeadura direta na recomposição vegetal de áreas ciliares: efeitos da sazonalidade, uso do solo, exclusão da predação, e profundidade na sobrevivência inicial. Semina (Ciências Agrárias) 26: 449-454.

Martins, S.V. 2001. Recuperação de Matas Ciliares. Editora Centro de Produções Técnicas, Viçosa.

Mattei, V.L. 1997. Avaliação de protetores físicos em semeadura direta de Pinus taeda L. Ciência Florestal 7: 91-100.

Meneghello, G.E. \& Mattei, V.L. 2004. Semeadura direta de timbaúva (Enterolobium contortisiliquum) e cedro (Cedrela fissilis) em campos abandonados. Ciência Florestal 14: 21-21.

Missanjo, E., Maya, C., Kapira, D., Banda, H. \& Kamanga-Thole, G. 2013. Effect of Seed Size and Pretreatment Methods on Germination of Albizia lebbeck. ISRN Botany 13: 1-4.

Moles, A.T. \& Westoby, M. 2004. Seedling survival and seed size: a synthesis of the literature. Journal of Ecology 92: 372-383.

Muller-Landau, H.C. 2010. The tolerance-fecundity trade-off and the maintenance of diversity in seed size. Proceedings of National Academy of Sciences 107: 4242-4247.

Obeso, J.R., Martínez, I. \& García, D. 2011. Seed size is heterogeneously distributed among destination habitats in animal dispersed plants. Basic and Applied Ecology 12: 134-140.

Oliveira, P.E., Gomes R.A., Sanches R.C. \& Saad, R.A. 2008. Análise da evolução da paisagem no entorno da represa dos rios Jaguari e Jacareí, estado de São Paulo, com base em sensoriamento remoto e sig. Geociências 27: 527-539.

Parrotta, J.A., Knowles, O.H. \& Wunderle, J.M. 1997. Development of floristic diversity in 10 -year-old restoration forests on a bauxite mined site in Amazonia. Forest Ecology and Management 99: 21-24.

Pereira, S.R. 2012. Recuperação Florestal através da semeadura direta: uso da superação de dormência e influência do tamanho das sementes e de gramíneas exóticas no estabelecimento de espécies de árvores. Tese de Doutorado, Universidade Federal de São Carlos, São Carlos. 
Queiroz, D.S., Salgado, L.T. \& Fernandes, L.O. 2008. Recuperação de pastagens degradadas. Informe Agropecuário 29: 55-65.

Ray, G.J. \& Brown, B.J. 1995. Dry forests: evaluation of tree propagation techniques. Restoration Ecology 3: 86-94.

Rodrigues, R.R., Lima, R.A.F., Gandolfi, S. \& Nave, A.G. 2009. On the restoration of high diversity forests: 30 years of experiences in the Brazilian Atlantic Forest. Biological Conservation 142: 1242-1251.

Rolim, G.S., Jesus, M.R. \& Nascimento, M.E.H. 2007. Restauração experimental de uma pastagem na mata atlântica através de semeadura direta. In: L.F.T. Menezes, F.R. Pires, O.J. Pereira (eds.). Ecossistemas Costeiros do Espírito Santo, EDUFES, Espirito Santo pp. 269-290.

Ruiz-Jaen, M.C. \& Aide, T.M. 2005. Restoration success: how is it being measured? Restoration Ecology 13: 569-577.

Sampaio, A.B. 2007. Regeneration of seasonal deciduous forest tree species in long- used pastures in central Brazil. Biotropica 39: 655-659.

Santos Junior, N.A. 2000. Estabelecimento inicial de espécies florestais nativas em sistema de semeadura direta. Dissertação de Mestrado, Universidade Federal de Lavras, Lavras.

Santos Júnior, N.A., Botelho, S.A., Davide, A.C. 2004. Estudo da germinação e sobrevivência de espécies arbóreas em sistema de semeadura direta, visando à recomposição de mata ciliar. Cerne 10: 103-117.

Santos, P.L., Ferreira L.A., Aragão, A.G., Amaral, L.A., Oliveira, A.S. 2012. Estabelecimento de espécies florestais nativas por meio de semeadura direta para a recuperação de áreas degradadas. Revista Árvore 36: 237-245.

Silva, J.B.C., Santos, P.E.C., Nascimento, W.M. 2002. Desempenho de sementes peletizadas de alface em função do material cimentante e da temperatura de secagem dos péletes. Horticultura Brasileira 20: 67-70.
Smith, P.T. \& Cobb, B.G. 1991. Accelerated germination of pepper seed by priming with salt solutions and water. HortScience 26: 417-419.

Sovu, P.S., Tigabu, M. \& Odén, P.C. 2010. Restoration of Former Grazing Lands in the Highlands of Laos Using Direct Seeding of Four Native Tree Species. Mountain Research and Development 30: 232-243.

Sun, D., Dickinson, G.R., Bragg, A.L. 1995. Direct seeding of Alphitonia petriei (Rhamnaceae) for gully revegetation in tropical northern Australia. Forest Ecology and Management 73: 249-257.

Tewari, B., Tewari, A., Shah, S., Pande, N. \& Singh, R.P. 2011. Physical attributes as indicator of seed maturity and germination enhancement in Himalayan Wild Cherry (Prunus cerasoides D. Don.). New Forests 41: 139-146.

Tunjai, P. \& Elliott, S. 2012. Effects of seed traits on the success of direct seedingfor restoring southern Thailand's lowland evergreenforest ecosystem. New Forests 43: 319-333.

Vázquez, C., Orozco, A., Rojas, M., Sánchez, M. \& Cervantes, V. 1997. La reproducción de las plantas: semillas y meristemos. Fondo de Cultura Económica, México D.F.

Vieira, D.L.M. \& Scariot, A. 2006. Principles of natural regeneration of tropical dry forests for restoration. Restoration Ecology 14: 11-20.

Woods, K. \& Elliott, S. 2004. Direct seeding for forest restoration on abandoned agricultural land in northern Thailand. Journal of Tropical Forest Science 16: 248-259.

Wright, S.J., Kitajima, K., Kraft, N.J.B., Reich, P.B., Wright, I.J, Bunker, D.E., Condit, R., Dalling, J.W., Davies, S.J., Díaz, S., Engelbrecht, B.M.J., Harms, K.E., Hubbell, S.P., Marks, C.O., Ruiz-Jaen, M.C., Salvador, C.M. \& Zanne, A.E. 2010. Functional traits and the growth-mortality trade-off in tropical trees. Ecology 91: 3664-3674.

Zar, J.H. 1996. Biostatistical analysis. Prentice-Hall International, London. 